Erratum

\title{
Correction: Ghasemi, M., et al. The Evaluation of Exogenous Application of Salicylic Acid on Physiological Characteristics, Proline and Essential Oil Content of Chamomile (Matricaria chamomilla L.) under Normal and Heat Stress Conditions. Agriculture 2016, 6, 31
}

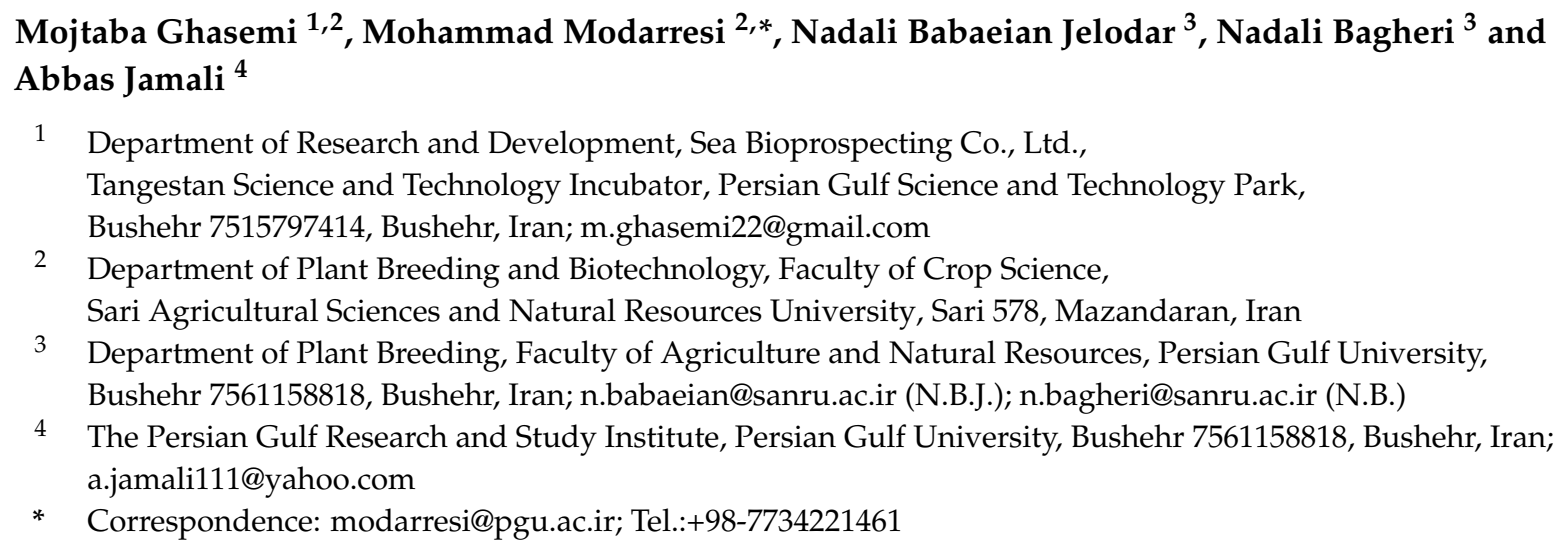

Academic Editor: Nieves Goicoechea

Received: 21 February 2017; Accepted: 21 February 2017; Published: 23 February 2017

The authors wish to make the following corrections to their paper [1]. The chamomile scientific name should be Matricaria chamomilla instead of Matricaria chamomila in the title and body text of this paper. In addition, the authors wish to remove Affiliation 5 (Young Researchers and Elite Club, Bushehr Branch, Islamic Azad University, Bushehr 751961955, Iran). Affiliation 1 has been changed from 'Tangestan Growth and Technology Center' to 'Tangestan Science and Technology Incubator'.

The authors would like to apologize for any inconvenience caused to the readers by these changes. The manuscript will be updated and the original will remain online on the article webpage.

\section{Reference}

1. Ghasemi, M.; Modarresi, M.; Babaeian Jelodar, N.; Bagheri, N.; Jamali, A. The Evaluation of Exogenous Application of Salicylic Acid on Physiological Characteristics, Proline and Essential Oil Content of Chamomile (Matricaria chamomilla L.) under Normal and Heat Stress Conditions. Agriculture 2016, 6, 31. [CrossRef]

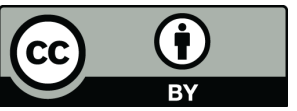

(C) 2017 by the authors. Licensee MDPI, Basel, Switzerland. This article is an open access article distributed under the terms and conditions of the Creative Commons Attribution (CC BY) license (http:/ / creativecommons.org/licenses/by/4.0/). 